CERN-PH-EP/2004-056

29 October 2004

\title{
Measurement of fragmentation properties of charmed particle production in charged-current neutrino interactions
}

\author{
CHORUS Collaboration
}

\begin{abstract}
During the years 1994-97, the emulsion target of the CHORUS detector was exposed to the wideband neutrino beam of the CERN SPS. In total about 100000 charged-current neutrino interactions were located in the nuclear emulsion target and fully reconstructed. From this sample of events which was based on the data acquired by new automatic scanning systems, $1048 \mathrm{D}^{0}$ events were selected by a pattern recognition program. They were confirmed as neutral-particle decays through visual inspection. Fragmentation properties of deep-inelastic charm production were measured using these events. Distributions of the $\mathrm{D}^{0}$ momentum, Feynman $x\left(x_{F}\right), z$ and $\tan \theta^{\text {out }}$, the transverse angle out of the leptonic plane defined by the muon and the neutrino, are presented. The mean value of $z$ was measured to be $\langle z\rangle=0.63 \pm 0.03$ (stat) \pm 0.01 (syst). From fits to the $z$ distribution, values for the Peterson parameter $\epsilon_{P}=0.108 \pm 0.017$ (stat) \pm 0.013 (syst) and the Collins-Spiller parameter $\epsilon_{C S}=0.21_{-0.04}^{+0.05}$ (stat) \pm 0.04 (syst) are found. For the average value of $x_{F}$ we find $\left\langle x_{F}\right\rangle=0.38 \pm 0.04$ (stat) \pm 0.03 (syst) and for the forward-backward asymmetry $A=0.79 \pm 0.14$ (stat) \pm 0.05 (syst). The distribution of $\tan \theta^{\text {out }}$ was measured with an average value $\left\langle\tan \theta^{\text {out }}\right\rangle=0.030 \pm 0.002$.
\end{abstract}

To be published in Physics Letters B 


\title{
CHORUS Collaboration
}

\author{
G. Önengüt \\ Çukurova University, Adana, Turkey
}

R. van Dantzig, M. de Jong, O. Melzer, R.G.C. Oldeman ${ }^{1}$, E. Pesen, J.L. Visschers

NIKHEF, Amsterdam, The Netherlands

M. Güler, U. Köse, M. Serin-Zeyrek, R. Sever, P. Tolun, M.T. Zeyrek

METU, Ankara, Turkey

M.G. Catanesi, M. De Serio, M. Ieva, M.T. Muciaccia, E. Radicioni, S. Simone

Università di Bari and INFN, Bari, Italy

A. Bülte, K. Winter

Humboldt Universität, Berlin, Germany ${ }^{2}$

B. Van de Vyver $^{3,4}$, P. Vilain ${ }^{5}$, G. Wilquet ${ }^{5}$

Inter-University Institute for High Energies (ULB-VUB) Brussels, Belgium

B. Saitta

Università di Cagliari and INFN, Cagliari, Italy

E. Di Capua

Università di Ferrara and INFN, Ferrara, Italy

S. Ogawa, H. Shibuya

Toho University, Funabashi, Japan

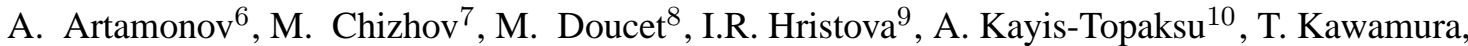
D. Kolev ${ }^{7}$, H. Meinhard, J. Panman, I.M. Papadopoulos, S. Ricciardi ${ }^{11}$, A. Rozanov ${ }^{12}$, R. Tsenov ${ }^{7}$, J.W.E. Uiterwijk, P. Zucchelli ${ }^{3,13}$

CERN, Geneva, Switzerland

J. Goldberg

Technion, Haifa, Israel

M. Chikawa

Kinki University, Higashiosaka, Japan

E. Arik

Bogazici University, Istanbul, Turkey

J.S. Song, C.S. Yoon

Gyeongsang National University, Jinju, Korea

K. Kodama, N. Ushida

Aichi University of Education, Kariya, Japan

S. Aoki, T. Hara

Kobe University, Kobe, Japan

T. Delbar, D. Favart, G. Grégoire, S. Kalinin, I. Makhlioueva

Université Catholique de Louvain, Louvain-la-Neuve, Belgium

P. Gorbunov, V. Khovansky, V. Shamanov, I. Tsukerman

Institute for Theoretical and Experimental Physics, Moscow, Russian Federation

N. Bruski, D. Frekers

Westfälische Wilhelms-Universität, Münster, Germany ${ }^{2}$

K. Hoshino, J. Kawada, M. Komatsu, M. Miyanishi, M. Nakamura, T. Nakano, K. Narita, K. Niu,

K. Niwa, N. Nonaka, O. Sato, T. Toshito

Nagoya University, Nagoya, Japan

S. Buontempo, A.G. Cocco, N. D’Ambrosio, G. De Lellis, G. De Rosa, F. Di Capua, A. Ereditato, G. Fiorillo, A. Marotta, M. Messina, P. Migliozzi, C. Pistillo, L. Scotto Lavina, P. Strolin, V. Tioukov

Università Federico II and INFN, Naples, Italy

K. Nakamura, T. Okusawa

Osaka City University, Osaka, Japan

U. Dore, P.F. Loverre, L. Ludovici, P. Righini, G. Rosa, R. Santacesaria, A. Satta, F.R. Spada

Università La Sapienza and INFN, Rome, Italy 
E. Barbuto, C. Bozza, G. Grella, G. Romano, C. Sirignano, S. Sorrentino

Università di Salerno and INFN, Salerno, Italy

Y. Sato, I. Tezuka

Utsunomiya University, Utsunomiya, Japan

\footnotetext{
${ }^{1}$ Now at Università La Sapienza, Rome, Italy. 7MS12P.

${ }^{3}$ Now at SpinX Technologies, Geneva, Switzerland.

${ }^{4}$ Fonds voor Wetenschappelijk Onderzoek, Belgium.

${ }^{5}$ Fonds National de la Recherche Scientifique, Belgium.

${ }^{6}$ On leave of absence from ITEP, Moscow.

${ }^{7}$ On leave of absence and at St. Kliment Ohridski University of Sofia, Bulgaria.

${ }^{8}$ Now at University of Maryland, MD, USA.

${ }^{9}$ Now at DESY, Hamburg.

${ }^{10}$ On leave of absence from Çukurova University, Adana, Turkey.

${ }^{11}$ Now at Royal Holloway College, University of London, Egham, UK.

${ }^{12}$ Now at CPPM CNRS-IN2P3, Marseille, France.

${ }^{13}$ On leave of absence from INFN, Ferrara, Italy.
}

${ }^{2}$ Supported by the German Bundesministerium für Bildung und Forschung under contract numbers 05 6BU11P and 05 
Introduction

Several experiments have presented results on production characteristics and fragmentation variables of charmed particles in charged-current (CC) interactions using different techniques [1]-[8].

The measurement of these fragmentation properties of charmed-particle production gives insight in the hadronization process and allows a better description of charm production to be formulated. In addition to the interest of the process per se, an improved knowledge helps to better understand the backgrounds in oscillation experiments that search for $\tau$ production or for the production of muons of a charge opposite to the one expected from the neutrino flavour of the beam.

With the exception of the E531 [6] emulsion experiment, the BEBC [5] bubble chamber and the NOMAD [7] electronic detector, results have been obtained based on opposite-charge two-muon (dimuon) data. In this kind of experiment the leading muon is interpreted as originating from the neutrino interaction vertex and the second one as the decay daughter of a charmed particle. Although the massive high-density detectors obtained large statistics, this method has some drawbacks. Only the decay muon of the charmed parent is seen, imposing an event sample composed of a mixture of charmedparticle species weighted by their muonic branching ratio. In addition, common to these experiments is the relatively high momentum threshold which has to be applied to the decay muon, so that only the high-momentum tail of the muon spectrum is accessible. In spite of this high threshold they all suffer from the presence of background from pion and kaon decays.

Unlike the dimuon experiments, the BEBC and NOMAD measurements were performed recognizing specific charm decay modes by reconstructing the invariant mass of the decay daughters. This method thus selects only a few specific decay modes, and therefore specific parent particle types. Up to now only the E531 experiment could measure fragmentation properties based on an inclusive charm-production sample.

Emulsion experiments like E531 and CHORUS study charm production and their characteristics by looking at the decay topology of the charmed hadron with high spatial resolution. The disadvantage of low statistics in this kind of experiment has been solved recently in the CHORUS experiment by developing the 'NetScan' technique [9] first used for the DONUT experiment [10]. A high-statistics sample of charm decays has been collected thanks to this technique.

In the present study, a large and clean sample of $\mathrm{D}^{0}$ 's was obtained. The momentum of the parent $\mathrm{D}^{0}$ cannot be directly measured in this experiment. Therefore, the momentum spectrum is obtained statistically from the average emission angle of the decay daughters making use of an unfolding procedure.

We report here on measurements of the $\mathrm{D}^{0}$ momentum distribution and the distributions of the fragmentation variables $x_{F}$ (Feynman $x$ ), $z$ (the ratio of the $\mathrm{D}^{0}$ energy and the energy transfer to the hadronic system) and $\tan \theta^{\text {out }}$ (the charm-production angle out of the leptonic plane).

The $\mathrm{D}^{0}$ 's form a mixture of directly produced $\mathrm{D}^{0}$ 's and $\mathrm{D}^{0}$ 's from the decays of the $\mathrm{D}^{*+}$ or $\mathrm{D}^{* 0}$. Thus, to determine the fragmentation functions of the originally produced charmed particle, this has to be taken into account. The measurements of the $x_{F}$ and $z$ distributions reported here are corrected to reflect the momentum of the originally produced charm particle.

\section{The experimental set-up}

The CHORUS detector is a hybrid set-up which combines a nuclear emulsion target with various electronic detectors such as trigger hodoscopes, a scintillating fibre tracker system, a hadron spectrometer, electromagnetic and hadronic calorimeters and a muon spectrometer [11]. The hadron spectrometer measures the bending of charged particles using an air-core magnet, the calorimeter is used to determine the energy of showers, and the muon spectrometer determines the charge and momentum of muons. Tracks reconstructed in the scintillating fibre detectors are followed upstream in the beam direction to locate the interaction vertices in the emulsion target. The nuclear emulsion is used as a target for neutrino interactions and to visualize a precise three-dimensional reconstruction of the vertex region of the events.

Although the experiment was originally designed to search for $\nu_{\mu} \rightarrow \nu_{\tau}$ oscillation by detecting the characteristic decay topology of the $\tau$ lepton produced in $\nu_{\tau}$ charged-current interactions, it is also possible to study charm particles which have a lifetime similar to that of the $\tau$ lepton.

The emulsion target has an overall mass of $770 \mathrm{~kg}$ and is segmented into four stacks. Each stack consists of eight modules of 36 plates of size of $36 \times 72 \mathrm{~cm}^{2}$. Each plate has a $90 \mu \mathrm{m}$ plastic base coated 
on both sides with a $350 \mu \mathrm{m}$ emulsion layer. Each stack is followed by three interface emulsion sheets having a $90 \mu \mathrm{m}$ emulsion layer on both sides of a $800 \mu \mathrm{m}$ thick plastic base and by a set of scintillating fibre target-tracker planes. The interface emulsion sheets and the fibre tracker system provide accurate particle trajectory predictions with a precision of about $150 \mu \mathrm{m}$ in position and $2 \mathrm{mrad}$ in the track angle.

The emulsion scanning is performed by computer-controlled, fully automatic microscopes equipped with a CCD camera and a read-out system called 'track selector' [12]. In order to recognize track segments in the emulsion, a series of tomographic images are taken by focusing at different depths in the emulsion thickness. The digitized images are shifted according to the predicted track angle and then added. The presence of aligned grains forming a track is detected as a local peak of the grey-level of the summed image. In the absence of an angular prediction, all angles smaller than $400 \mathrm{mrad}$ are tried by parallel processing hardware. The track-finding efficiency of the track selector is higher than $98 \%$ for track slopes less than $400 \mathrm{mrad}$ [13].

The CHORUS detector was exposed to the wide-band neutrino beam of the CERN SPS during the years 1994-97. The beam consisted mainly of $\nu_{\mu}$ with a contamination of 5\% $\bar{\nu}_{\mu}$ and about $1 \% \nu_{e}$. In total $\approx 94000 \nu_{\mu}$ CC events with a negative muon measured in the spectrometer were located and fully reconstructed in the emulsion target. In order to identify charm decays, a volume of $1.5 \mathrm{~mm} \times 1.5 \mathrm{~mm} \times 6.3 \mathrm{~mm}$ around the located vertex position was scanned with a very fast scanning system and all tracks in that volume were reconstructed. A detailed description of the NetScan procedure is given in Ref. [14].

\section{Experimental procedure}

The selection and identification of the $\mathrm{D}^{0}$ sample used in this analysis was based purely on the decay topology of the $\mathrm{D}^{0}$. First, all tracks were reconstructed in the NetScan volume and only those which originated inside the volume were retained. Then, vertices were defined by combining tracks on the basis of their minimum distance. Out of these emulsion tracks those which could be matched with a fibre-tracker track (TT track) were selected as matched tracks. The primary vertex was defined as the one which contained the TT track recognized as the primary muon of the CC interaction. Charm candidates were selected by requiring an additional vertex in the event. The procedure is described in detail in Ref. [13].

These selection criteria were applied to the full sample of located CC interactions. In total about 3000 events were selected for visual inspection to confirm the decay topology in the emulsion. In the visual inspection, a vertex found in the emulsion was tagged as a decay vertex if it was downstream of the primary neutrino interaction vertex and if no other activity (nuclear break-up or Auger electron) at the decay point was observed. For this analysis only those events were used in which both the primary and decay vertices were reconstructed and where the secondary vertex was consistent with the decay of a neutral particle. After the visual inspection $1048 \mathrm{D}^{0}$ events were confirmed out of the selected events. Out of these $\mathrm{D}^{0}$ events 819 show a two-prong decay topology, 226 a four-prong topology, and only three have six charged daughter tracks at the decay vertex [15]. The majority of the candidates which were not confirmed consists mostly of events with additional low-momentum tracks with a large multiple scattering, delta rays, and gamma conversions.

The efficiency of the charm selection was evaluated with a GEANT3 [16] based Monte Carlo simulation of the experiment. Large samples of events with charm production in deep-inelastic neutrino interactions were generated according to the beam spectrum using a generator (JETTA) [17] which is based on LEPTO [18] and JETSET [19]. The simulated response of the electronic detectors was processed through the same analysis chain. To evaluate the NetScan efficiency, realistic conditions of track densities in the emulsion needed to be reproduced. To achieve this, the emulsion data of simulated events were merged with real NetScan data where the volume did not contain any event but only tracks which stop or pass through the volume. The combined data were passed through the same NetScan reconstruction and selection programs as used for the real data.

Possible background sources for $\mathrm{D}^{0}$ decays are mainly electron-positron pairs and decays of strange particles. An electron-positron pair can be tagged as a $\mathrm{D}^{0}$ decay if the opening angle is large. Since the momentum of these electrons is very low in this case, at least one of them is scattered within a few plates downstream of the decay vertex. So, this background is eliminated by following the decay 


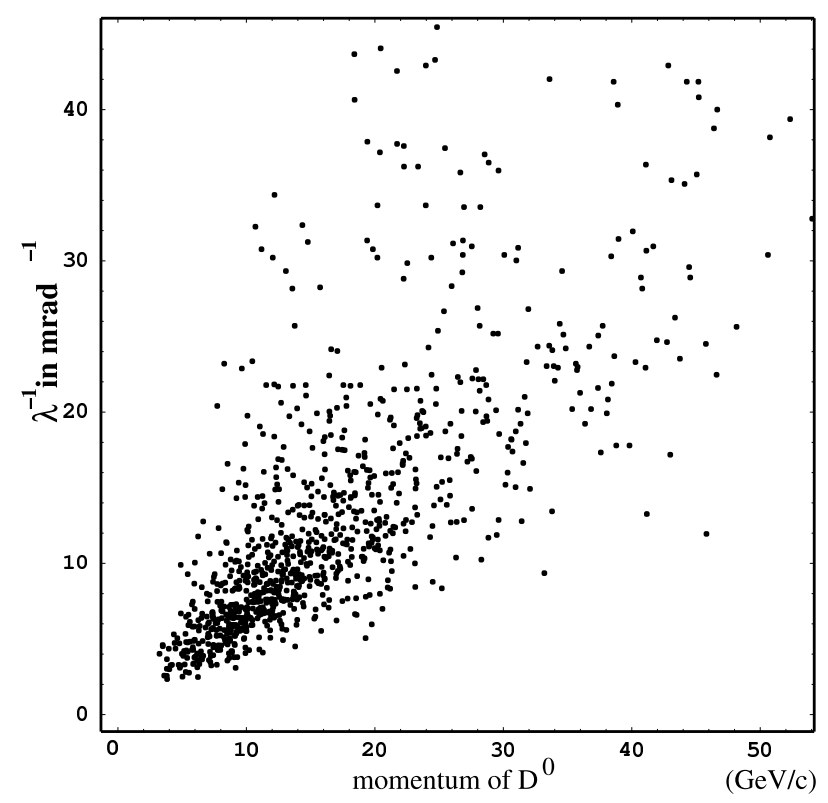

Figure 1: Correlation between $\mathrm{D}^{0}$ momentum and the inverse of the geometrical average of the angles of daughter tracks with respect to the parent

daughter which does not match a TT track. The background coming from neutral strange particle decays such as $\Lambda^{0}$ and $\mathrm{K}_{s}^{0}$ is negligible for this analysis owing to their much longer decay length. Their number has been evaluated to be $11.5 \pm 1.9 \Lambda^{0}$ 's and $25.1 \pm 2.9 \mathrm{~K}_{s}^{0}$ 's in the full $\mathrm{D}^{0}$ sample [20], respectively.

\section{$4 \quad$ Analysis and results}

To study the fragmentation of charmed quarks into hadrons one usually defines the ratio $z$ of the energy of the charmed particle $E^{D}$ and the energy transfer to the hadronic system $\nu$ as

$$
z=E^{D} / \nu .
$$

The estimate of $\nu$ is obtained from the measurement of the total energy deposited in the calorimeter, after subtraction of the energy loss of the muon and correction for the energy deposited in the emulsion stacks. The energy resolution for hadronic showers has been measured in charged-particle beams and is [11]

$$
\frac{\sigma(E)}{E}=\frac{0.323 \pm 0.024}{\sqrt{E / \mathrm{GeV}}}+(0.014 \pm 0.007)
$$

The momentum of the $\mathrm{D}^{0}$, required to define $z$, is not directly measured. Instead, one can exploit the correlation between the momentum and the angular distribution of the decay products. These angles are measured very precisely. For a given decay mode this correlation is determined by the decay kinematics and is model-independent. This method inferring the momentum of parent particles in emulsion experiments was first described in Ref. [21]. The most sensitive parameter is the geometrical average of the angle of the decay daughters with respect to the direction of the $\mathrm{D}^{0}, \lambda$, which is inversely proportional to the momentum of the parent, $p_{D}$. Figure 1 shows the correlation of $\lambda^{-1}$ and $p_{D}$ from a simulation of the mixture of the various decay modes of the $\mathrm{D}^{0}$ with the JETTA generator. The correlation is clear, although no precise measurement of $p_{D}$ can be obtained on an event-by-event basis. However, the correlation can be exploited to determine the momentum distribution from the $\lambda$ distribution on a statistical basis by making use of an unfolding procedure.

Several methods are used to obtain measurements of the angles of the tracks in the emulsion plates. During the manual checking of the selected events, the parameters of all tracks from the decay vertex are measured and the coordinates of the vertices are determined. The most precise measurement of the direction of the charged decay-daughters is determined by a fit to the NetScan track segments in the emulsion plates. The average angular resolution for the decay products of the $\mathrm{D}^{0}$ with the latter method 


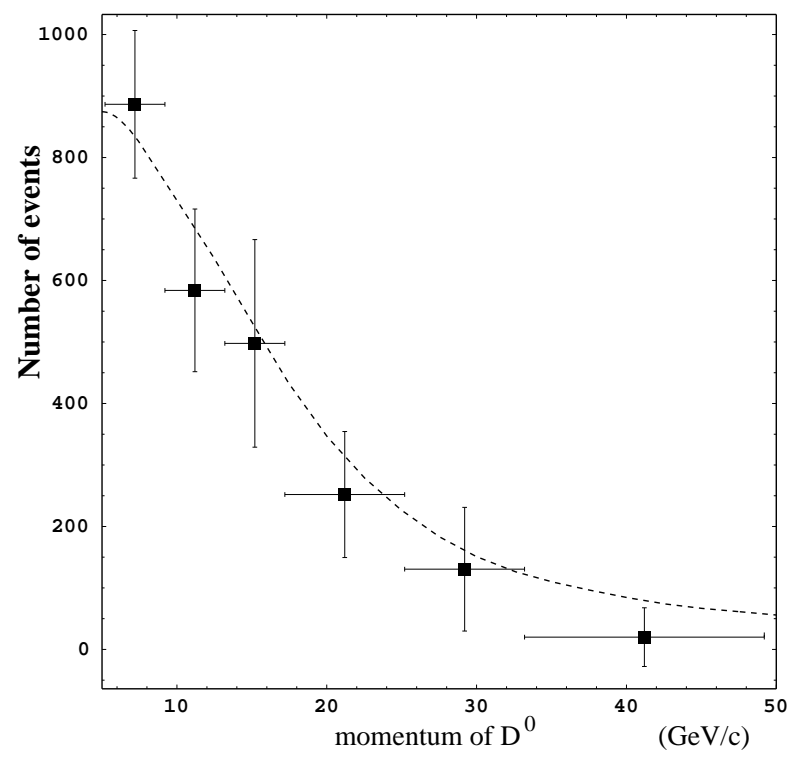

Figure 2: Momentum distribution of $\mathrm{D}^{0}$ 's produced inclusively in this experiment. The dashed curve shows the prediction of the JETTA model quoted in the text. The data points indicate the central value of the result, the vertical error bars mark the square root of the diagonal elements of the covariance matrix, while the horizontal bars show the width of the bins. The correlation coefficient between neighbouring bins is $\approx-0.5$.

is $1 \mathrm{mrad}$. The $\mathrm{D}^{0}$ direction is found by connecting the position of the primary interaction vertex and the decay vertex. The angular resolution obtained for the $\mathrm{D}^{0}$ is better than $3 \mathrm{mrad}$ for decay lengths larger than $300 \mu \mathrm{m}$. This high-resolution method requires the reconstruction of at least two tracks at the primary vertex and at the decay vertex in the NetScan procedure. The latter requirement introduces a loss of efficiency of $30 \%$ and is used only for the measurements of $x_{F}$ and $\tan \theta^{\text {out }}$. In these cases also an extra requirement on the length of the decay path of the $\mathrm{D}^{0}$ was introduced, depending on the resolution required for the measurement: $40 \mu \mathrm{m}$ for the $x_{F}$ measurement and $300 \mu \mathrm{m}$ for the $\tan \theta^{\text {out }}$ measurement. Since $\tan \theta^{\text {out }}$ requires a good definition of the direction of the muon perpendicular to the beam, an additional cut on the opening angle of the muon, $\theta_{\mu}>30 \mathrm{mrad}$, was applied.

For the measurement of the momentum and $z$ distribution where the resolution in the direction of the $\mathrm{D}^{0}$ is not critical, no cuts on the decay path of the $\mathrm{D}^{0}$ are applied. The interaction vertex and the decay vertex as obtained by the manual scanning procedure were used. This measurement is available for all events.

In order to unfold the measured distribution, the assumption is made that the probability density function in the physical parameter ('physical distribution') can be approximated by a linear superposition of cubic B-splines. With this choice of basis, no physical assumption is made other than the smoothness of the behaviour of the physical function over an interval comparable to the knot distance of the B-splines. The resolution of the measurements is evaluated using the simulation and expressed as a smearing matrix, while the acceptance and efficiency are taken into account by the normalization of this matrix. The coefficients of the B-splines are determined by finding the best fit to the distribution in the measured variable(s). For presentation of the results, the function formed by the superposition of splines is re-binned into a histogram. The covariance matrices are found by straightforward error propagation. The unfolding procedure corrects for the overall experimental efficiency; the normalization of all distributions reflects the total number of $\mathrm{D}^{0}$ 's produced in the emulsion target. A similar procedure is described in Ref. [22], although for the present study the algorithm had to be newly implemented in Mathematica ${ }^{T M}$ [23]. The unfolding procedure was tested on simulated data. It was found that the results are unbiased and that correct error estimates are evaluated. The validity of the method has also been checked by using simulated input data with a different distribution in the physical variable as the one used to calculate the smearing matrix. Also in this case the results reproduced the physical distribution of the simulated input data. 

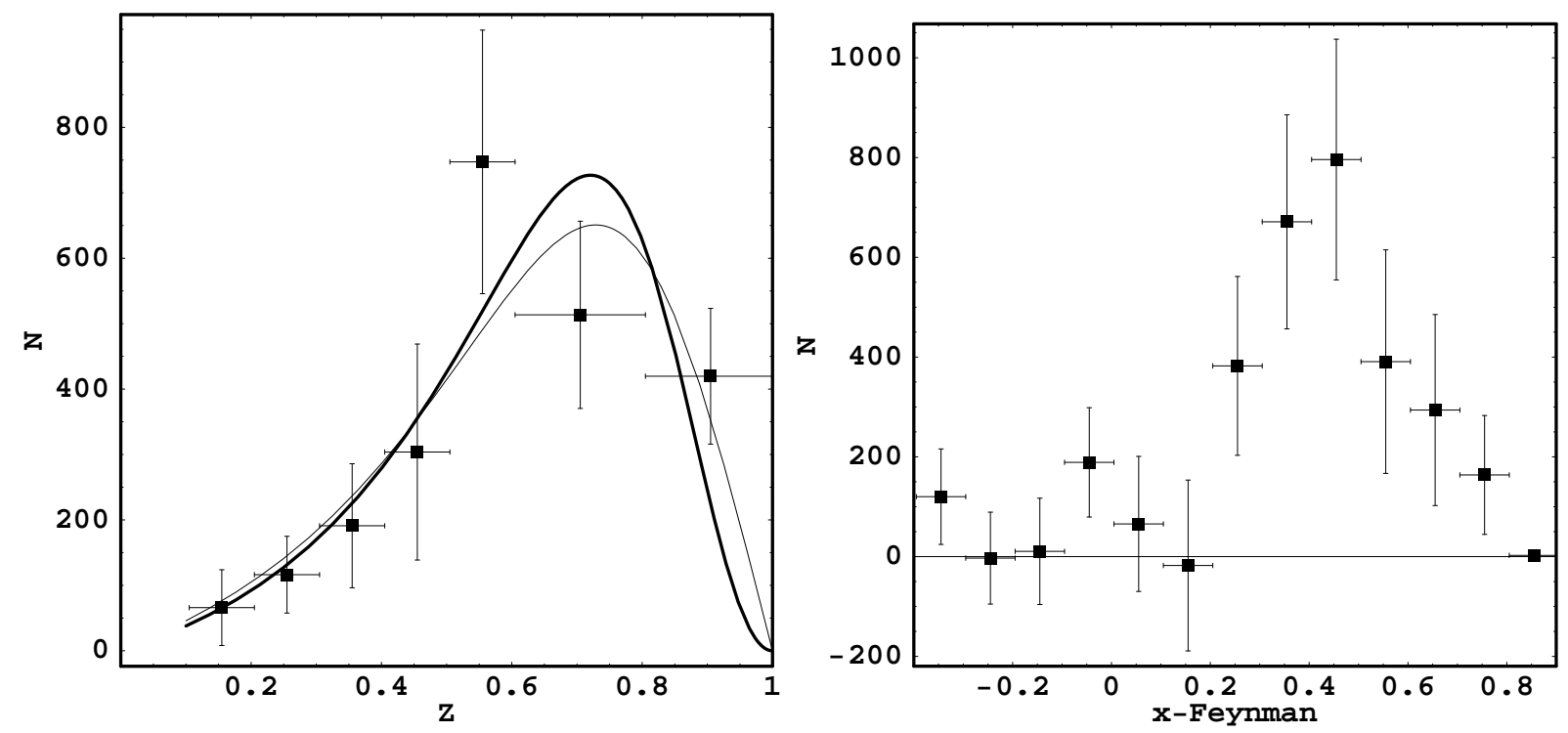

Figure 3: Measured distribution of $z$ (left panel) and $x_{F}$ (right panel) of the $\mathrm{D}^{0}$ corrected to represent the production of the primary charmed particle using the Lund model. The data points indicate the central value of the result, the vertical error bars mark the square root of the diagonal elements of the covariance matrix, while the horizontal bars show the width of the bins. In $z$, the correlation coefficient between neighbouring bins ranges between $\approx-0.1$ at small $z$ and $\approx-0.6$ at $z=1$, while in $x_{F}$ the coefficient ranges between $\approx-0.1$ and $\approx+0.4$. No correction for Fermi motion is applied. In the left panel the thicker curve represents the fit to the Peterson et al. model, while the thinner one shows the fit to the Collins-Spiller model.

The measurement of the momentum distribution of the $\mathrm{D}^{0}$ as obtained with this unfolding procedure is shown in Fig. 2. The unfolding method uses the measured distribution of $1 / \lambda$ as input. The measured distribution is compatible with the distribution predicted by the Monte Carlo model. Since the detection efficiency for $\mathrm{D}^{0}$ 's depends mainly on the momentum distribution, the consistency between the model and the data supports the efficiency calculations used in other CHORUS publications $[14,15]$.

The most sensitive method of measuring the $z$ distribution is to unfold the two-dimensional distribution in the $\left(\lambda^{-1}, \nu\right)$ plane. Technically, the one-dimensional $z$ distribution is approximated by a superposition of B-splines, while the coefficients of the spline-functions are found by obtaining the best agreement of the superposition of the corresponding simulated distributions with the measured twodimensional distribution in $\lambda^{-1}$ and $\nu$. Figure 3 shows the $z$ distribution of all $\mathrm{D}^{0}$ 's in the final state. The $z$ distribution is corrected to reflect the production of the charmed particle at the primary vertex. For this correction the Lund model [19], which predicts that a large fraction (75\%) of the $\mathrm{D}^{0}$ 's is produced as decay product of the $\mathrm{D}^{*}$, is used. The systematic error introduced by this model dependence is calculated by applying the correction for an assumed range of the above fraction between $50 \%$ and $100 \%$ and is found to be $1 \%$ in the average value of $z$. The events predominantly populate the high- $z$ region. This is expected if most charmed particles appear as current fragments of the hadronic system. A common parametrization of the $z$ distribution is the formula of Peterson et al. [24]

$$
D_{p}^{h}(z)=\frac{N}{z\left(1-\frac{1}{z}-\epsilon_{P}(1-z)\right)^{2}}
$$

where $N$ is a normalization factor and $\epsilon_{P}$ is a free parameter to be determined from the data. The thicker curve in Fig. 3 represents the fit result with the value of $\epsilon_{P}=0.108 \pm 0.017 \pm 0.013$, the first error being statistical and the second one systematic. This convention will be used throughout the text. The fit to the 
data using the Collins-Spiller [25] approach with the parametrization

$$
D_{c}^{h}(z)=\frac{N\left(\frac{\epsilon_{C S}(2-z)}{1-z}+\frac{1-z}{z}\right)\left(1+z^{2}\right)}{\left(1-\frac{\epsilon_{C S}}{1-z}-\frac{1}{z}\right)^{2}},
$$

with $\epsilon_{C S}$ as a free parameter, gave a value for the fit parameter $\epsilon_{C S}=0.21_{-0.04}^{+0.05} \pm 0.04$. This fit is shown by a thin curve in the figure. The mean value was measured to be $\langle z\rangle=0.63 \pm 0.03 \pm 0.01$ consistent with previous measurements (see Section 5). The largest systematic error in the measurements of kinematic variables is given by the uncertainty of the absolute scale of the energy definition in the calorimeter $(\approx 5 \%)$. This uncertainty is included in the systematic error.

We have chosen to define $z$ as the ratio of the energy of the primary charmed particle produced and the energy transfer $\nu$. This choice has the advantage of involving two quantities which are in principle observable. If we perform the analysis within the Lund model where one chooses as variable $z^{S}$ the ratio of the energy of the primary charmed particle produced and the energy of the string, we find $\epsilon_{P}^{S}=$ $0.083 \pm 0.013 \pm 0.010$. In dimuon experiments it is customary to use another variable $z^{Q}$ defined as the ratio of the momentum of the primary charmed particle and the momentum of the charm quark produced in the interaction. When the analysis is performed using the latter convention a value of $\epsilon_{P}^{Q}=$ $0.059 \pm 0.010 \pm 0.008$ is obtained. It should be noted that both $\epsilon_{P}^{S}$ and $\epsilon_{P}^{Q}$ cannot be compared with $\epsilon_{P}$ and with each other since they are based on different definitions of $z$.

It is also customary to describe the fragmentation process in terms of the Feynman $x$ variable $\left(x_{F}\right)$, which is defined as the longitudinal momentum of the charmed particle in the hadronic centre-ofmomentum frame, divided by the maximum possible momentum for the particle. In terms of kinematic observables, it can be expressed as

$$
x_{F}=2 \gamma \frac{p_{L}^{D}-\beta E^{D}}{W}
$$

where $\gamma$ and $\beta$ are the usual relativistic variables ${ }^{1)}, W$ the invariant mass of the hadronic system, and $p_{L}^{D}$ and $E^{D}$ the longitudinal momentum and energy of the $\mathrm{D}^{0}$, respectively. In addition to the momentum and direction of the $\mathrm{D}^{0}$, the direction vector of the hadronic system transverse to the beam direction, $\overrightarrow{h_{T}}$, has also to be measured. The best estimate can be obtained by using the measurement of the muon direction, $\overrightarrow{\mu_{T}}$, and assuming transverse-momentum conservation:

$$
p_{h} \overrightarrow{h_{T}}=-p_{\mu} \overrightarrow{\mu_{T}}
$$

where $p_{\mu}$ is the absolute muon momentum, and $p_{h}$ the total hadronic momentum. The latter quantity is estimated using $\nu$ as defined above.

We define two observables $f_{1}=\left(\nu+M_{p}\right) \cos \alpha /\left(W^{2} \lambda\right)$ and $f_{2}=\sqrt{\nu^{2}+Q^{2}} /\left(W^{2} \lambda\right)$, where $\alpha$ is the angle of the $\mathrm{D}^{0}$ with respect to the hadronic system, $M_{p}$ is the nucleon mass, and $Q^{2}$ is the square of the four-momentum transferred from the incoming neutrino to the hadronic system. $W^{2}$ and $Q^{2}$ are calculated using the hadronic energy and the measurement of the muon momentum and angle. The two observables $f_{1}$ and $f_{2}$ have a good correlation with $x_{F}$. A two-dimensional unfolding procedure is performed in the plane $\left(f_{1}, f_{2}\right)$. Figure 3 shows the $x_{F}$ distribution of the $\mathrm{D}^{0}$ production. A correction similar to the one applied to the $z$ distribution using the Lund model was made, such that the $x_{F}$ distribution is valid for the originally produced charm particle. From the distribution one observes that most $\mathrm{D}^{0}$ 's are produced in the forward direction in the centre-of-momentum frame. For the average value we find $\left\langle x_{F}\right\rangle=0.38 \pm 0.04 \pm 0.03$. The forward-backward asymmetry, $A$, is found to be $A=0.79 \pm 0.14 \pm 0.05$ indicating once more that most charmed particles are produced in the forward region. The systematic error quoted in $\left\langle x_{F}\right\rangle$ and $A$ contains the uncertainty introduced by the fragmentation model when applying the correction for the fact that part of the $\mathrm{D}^{0}$ 's are decay products and the uncertainty of the energy scale of the calorimeter measurement. This error was evaluated by repeating the analysis using the same variation in the fraction of directly produced $\mathrm{D}^{0}$ 's compared to all $\mathrm{D}^{0}$ 's and the same variation in the energy scale of the calorimeter as the ones used to evaluate the systematic error for the $z$ measurement.

\footnotetext{
1) When the correction using the Lund model is made, the $\beta$ and $\gamma$ of the Lund string are used.
} 


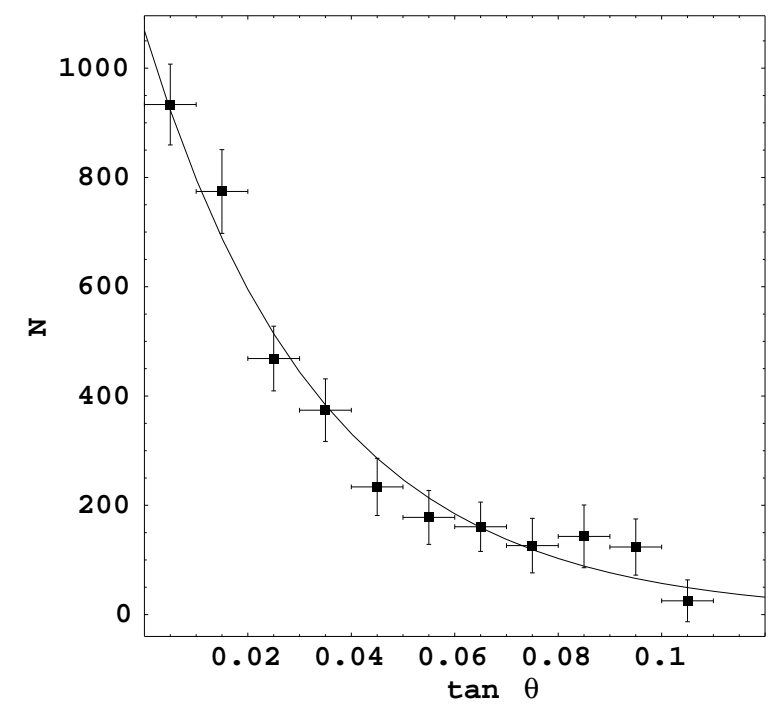

Figure 4: Measurement of the distribution of the $\mathrm{D}^{0}$ production angle out of the plane formed by the muon and the neutrino (lepton plane). The curve shows an exponential fit to the data as described in the text. The data points indicate the central value of the result, the vertical error bars mark the square root of the diagonal elements of the covariance matrix, while the horizontal bars show the width of the bins. The correlation coefficient between neighbouring bins is small $(-0.1<\rho<0.1)$.

Table 1: Results of the measurements of the fragmentation variables

\begin{tabular}{cccc}
\hline Variables & Mean value & Fit value & Asymmetry \\
\hline$z$ & $0.63 \pm 0.03 \pm 0.01$ & $\epsilon_{P}=0.108 \pm 0.017 \pm 0.013$ & - \\
& & $\epsilon_{C S}=0.21_{-0.04}^{+0.05} \pm 0.04$ & \\
\hline$\left\langle x_{F}\right\rangle$ & $0.38 \pm 0.04 \pm 0.03$ & - & $A=0.79 \pm 0.14 \pm 0.05$ \\
\hline$\left\langle\tan \theta^{\text {out }}\right\rangle$ & $0.030 \pm 0.002$ & $b=0.034 \pm 0.003$ & - \\
\hline
\end{tabular}

Often the transverse momentum $p_{T}$ with respect to the current-direction is also used to characterize the fragmentation process. However, the distribution of $p_{T}$ has a width similar to the resolution, and the measurement is dominated by systematic errors. The most precise measurement relevant to the transverse properties is the out-of-plane angle, $\theta^{\text {out }}$, the angle between the lepton plane formed by the muon and the neutrino and the $\mathrm{D}^{0}$. This observable is defined by direction measurements alone which are obtained with high precision. The resolution in $\tan \theta^{\text {out }}$ is 0.006 (RMS) for the selection criteria given above ( $\mathrm{D}^{0}$ decay path greater than $300 \mu \mathrm{m}$ and $\theta_{\mu}>30 \mathrm{mrad}$ ). Therefore, the unfolding procedure uses the measured distribution of $\tan \theta^{\text {out }}$ directly as input and the procedure is used here merely to correct for the resolution of the measurement.

Figure 4 shows the distribution of $\tan \theta^{\text {out }}$ for $\mathrm{D}^{0}$ production. An average value $\left\langle\tan \theta^{\text {out }}\right\rangle=$ $0.030 \pm 0.002$ was obtained. The systematic error of this result is negligible, since the precision of the direction measurements is very high in the emulsion. The distribution is given for the observed $\mathrm{D}^{0}$, irrespective of its origin. The distribution was neither corrected for the $\mathrm{D}^{*}$ decay into the $\mathrm{D}^{0}$ nor for Fermi motion. To ease the use of our data, a purely phenomenological description of the distribution is useful. An exponential function of the form $A e^{-\tan \theta^{\text {out }} / b}$ with the best-fit value $b=0.034 \pm 0.003$ provides a good approximation to the distribution. The curve representing this parametrization is shown in the figure.

The results of this study are summarized in Table 1 .

\section{$5 \quad$ Summary and discussion}

Using a high-statistics inclusive $\mathrm{D}^{0}$ sample obtained by selecting decay topologies in an emulsion target, a study of the fragmentation parameters was performed. Although the momentum of the $\mathrm{D}^{0}$ is 
Table 2: Comparison of results of the measurements of fragmentation variables. As explained in the text, the different choices of definition of $z$ influence the fit results for $\epsilon_{P}$ and $\epsilon_{C S}$. With the same choice of $z$ as used by NOMAD and the dimuon experiments, CHORUS would find $\epsilon_{P}^{Q}=0.059 \pm 0.010 \pm 0.008$.

\begin{tabular}{lccc}
\hline Experiment & $\langle z\rangle$ & $\epsilon_{P}$ and $\epsilon_{C S}$ & $\left\langle x_{F}\right\rangle$ and $A$ \\
\hline CDHS[2] & $0.68 \pm 0.08$ & $\epsilon_{P}^{Q}=[0.02,0.14]$ & - \\
\hline E531[6] & $0.59 \pm 0.04$ & $\epsilon_{P}=0.076 \pm 0.014$ & $A=0.620 \pm 0.092$ \\
\hline CCFR[4] & $0.56 \pm 0.03$ & $\epsilon_{P}=0.22 \pm 0.05$ & - \\
& & $\epsilon_{C S}=0.88 \pm 0.12$ & - \\
\hline BEBC[5] & $0.59 \pm 0.03 \pm 0.08$ & - & - \\
\hline CHARM II[3] & $0.66 \pm 0.03$ & $\epsilon_{P}^{Q}=0.072 \pm 0.017$ & \\
\hline NOMAD[7] & $0.67 \pm 0.02 \pm 0.02$ & $\epsilon_{P}^{Q}=0.075 \pm 0.028 \pm 0.036$ & $\left\langle x_{F}\right\rangle=0.47 \pm 0.05$ \\
& & $\epsilon_{C S}=0.13 \pm 0.08 \pm 0.11$ & - \\
\hline NuTeV[8] & - & $\epsilon_{C S}=2.07 \pm 0.31$ & \\
\hline CHORUS & $0.63 \pm 0.03 \pm 0.01$ & $\epsilon_{P}=0.108 \pm 0.017 \pm 0.013$ & $\left\langle x_{F}\right\rangle=0.38 \pm 0.04 \pm 0.03$ \\
& & $\epsilon_{C S}=0.21_{-0.04}^{+0.05} \pm 0.04$ & $A=0.79 \pm 0.14 \pm 0.05$ \\
\hline
\end{tabular}

not directly obtainable in this experiment, an unfolding procedure exploited the correlation between momentum and the opening angle of the decay products.

The momentum distribution predicted by the Lund model on which the JETTA generator is based is consistent with the measurement reported here. This supports the efficiency calculations used in other CHORUS publications.

Results obtained by other experiments are compared with the measurements presented here in Table 2. One should note, however, that this comparison is somewhat difficult for the values obtained from the $z$ distribution due to the different definitions of $z$ used by the various groups as pointed out in Section 4. Indeed, the table shows that the measured values of $\epsilon_{P}$ and $\epsilon_{C S}$ tend to vary considerably between the experiments. The variations introduced by the different definitions of $z$ were evaluated by repeating the analysis of our data with a variety of choices. In particular, if we apply the same definition, $z^{Q}$, as the one used by the dimuon experiments [2,3] and NOMAD [7] which were exposed to a beam of similar neutrino energy, we find a value $\epsilon_{P}^{Q}=0.059 \pm 0.010 \pm 0.008$, in good agreement with their results. The higher energy of the neutrino beam used by CCFR [4] and $\mathrm{NuTeV}$ [8] could also play a role in the comparison with these experiments.

In addition, the charmed-particle mixtures used for the measurements are not the same for each experiment. In this experiment all $\mathrm{D}^{0}$ 's (both directly produced and as decay product of the $\mathrm{D}^{*}$ ) are used while NOMAD used only the $\mathrm{D}^{*+}$ and E531 gave their results for all charged and neutral charmed particles. The dimuon experiments use a mixture according to the muonic branching ratio of the particle types. Moreover, they have to infer, for example, $z$ from the momentum spectrum of the decay muon. Due to the high momentum threshold which had to be applied in these experiments, only the high-momentum tail of the decay spectrum is exploited.

We find consistency among the measurements of $\left\langle x_{F}\right\rangle$ and the asymmetry in $x_{F}$.

\section{Acknowledgements}

We gratefully acknowledge the help and support of the neutrino beam staff and of the numerous technical collaborators who contributed to the detector construction, operation, emulsion pouring, development, and scanning. The experiment was made possible by grants from the Institut Interuniversitaire des Sciences Nucléaires and the Interuniversitair Instituut voor Kernwetenschappen (Belgium), the Israel Science Foundation (grant 328/94) and the Technion Vice President Fund for the Promotion of Research (Israel), CERN (Geneva, Switzerland), the German Bundesministerium für Bildung und Forschung (Germany), the Institute of Theoretical and Experimental Physics (Moscow, Russia), the Istituto Nazionale di Fisica Nucleare (Italy), the Promotion and Mutual Aid Corporation for Private Schools of Japan and Japan Society for the Promotion of Science (Japan), the Korea Research Foundation Grant (KRF-2003- 
005-C00014) (Republic of Korea), the Foundation for Fundamental Research on Matter FOM and the National Scientific Research Organization NWO (The Netherlands), and the Scientific and Technical Research Council of Turkey (Turkey). We gratefully acknowledge their support.

\section{References}

[1] M. Jonker et al., Phys. Lett. B107 (1981) 241.

[2] H. Abramowicz et al., Z. Phys. C15 (1982) 19.

[3] P. Vilain et al., Eur. Phys. J. C11 (1999) 19.

[4] S.A. Rabinowitz et al., Phys. Rev. Lett. 70 (1993) 134; M. Goncharov et al., Phys. Rev. D64 (2001) 112006.

[5] A. E. Asratyan et al., Z. Phys. C68 (1995) 43.

[6] N. Ushida et al., Phys. Lett. B206 (1988) 380.

[7] P. Astier et al., Phys. Lett. B526 (2002) 278.

[8] D. Naples et al., Nucl. Phys. (Proc. Suppl.) B118 (2003) 164.

[9] N. Nonaka, Ph.D. thesis, Nagoya University, Japan (2002).

[10] K. Kodama et al., Nucl. Instr. and Meth. A493 (2002) 45.

[11] E. Eskut et al., Nucl. Instr. and Meth. A401 (1997) 7.

[12] T. Nakano. Ph.D. thesis, Nagoya University, Japan, 1997.

[13] M. Güler, Ph.D. thesis, METU, Ankara, Turkey (2000).

[14] A. Kayis-Topaksu et al., Phys. Lett. B527 (2002) 173.

[15] CHORUS Collaboration, "Measurement of $\mathrm{D}^{0}$ production and decay in neutrino interactions", in preparation.

[16] GEANT 3.21, CERN program library long write up W5013.

[17] P. Zucchelli, Ph.D. thesis, University of Ferrara, Italy (1996).

[18] G. Ingelman, Preprint TSL/ISV 92-0065, Uppsala University, Sweden (1992).

[19] T. Sjöstrand, Comput. Phys. Commun. 82 (1994) 74.

[20] M. Sorrentino, CHORUS internal note 2000027 (2004), http://choruswww.cern.ch/Publications/Notes/charm_background.pdf

[21] S. Petrera and G. Romano, Nucl. Instrum. and Meth. 174 (1980) 61.

[22] V. Blobel, Unfolding methods in High Energy Physics, in Proceedings of the 1984 CERN School of Computing, CERN 85-02 (1985) and DESY 84-118;

V. Blobel, The RUN manual, OPAL Technical Note TN361, 1996.

[23] S. Wolfram, The Mathematica Book, 3rd ed. (Wolfram Media/Cambridge University Press, 1996).

[24] C. Peterson et al., Phys. Rev. D27 (1983) 105.

[25] P.D.B. Collins and T.P Spiller, J. Phys. G11 (1985) 1289. 